\title{
GLOBAL AND LOCAL ESTIMATES OF THE CURRENT SHEET THICKNESS: CDAW-6
}

\author{
T. I. Pulkkinen, * D. N. Baker,** D. G. Mitchell,*** \\ R. L. McPherron, $†$ C. Y. Huang $\ddagger$ and L. A. Frank $\ddagger$ \\ * Finnish Meteorological Institute, Box 503, SF-00101 Helsinki, Finland \\ ** NASAVGSFC, Code 690, Greenbelt, MD 20771, U.S.A. \\ *** JHU/APL, Laurel, MD 20723, U.S.A. \\ † IGPP/UCLA, Los Angeles, CA 90024, U.S.A. \\ $¥$ University of Iowa, Iowa City, IA 52242, U.S.A.
}

\begin{abstract}
The growth phase development of the near-Earth magnetotail configuration during the CDAW-6 substorm (22 March 1979, 1054 UT) is studied using two complementary methods. The global magnetic field configuration is modeled using data from two suitably located spacecraft and temporally evolving variations of the Tsyganenko magnetic field model. These results are compared with a local calculation of the current sheet location and thickness previously carried out by McPherron et al. /1/. The good agreement between the two methods provides a positive test for the accuracy of the global model. Both models indicate the formation of an extremely thin current sheet during the substorm growth phase. The plasma electron data together with the rate of pitch-angle scattering estimated from the global field model suggest that the onset may be initiated by the chaotization of electrons and consequent growth of the tearing instability.
\end{abstract}

\section{INTRODUCTION}

The plasma sheet in the nightside magnetotail is a region critically important for the dynamics of the magnetospheric system. Soon after the first spacecraft measurements from the inner magnetotail were made, this region was found to undergo significant changes both in the magnetic field configuration and topology as well as in the plasma distributions $/ 2 /$. The thinning of the nearEarth tail current sheet in association with geomagnetic activity has been established in several statistical and case studies. At geostationary orbit, particle dropouts and the stretching of the magnetic field have been observed to precede the onset of substorms $/ 3 /$. In the mid-tail region tailward of $\sim 15 R_{E}$, the plasma sheet thins only after the expansion phase onset, often in connection with fast tailward plasma flows $/ 4,5,6 /$.

The detailed time development of the collisionless plasma sheet during substorms is one of the most critical questions in modeling the plasma processes during the substorm cycle. The tearing instability has been suggested to initiate magnetic reconnection at substorm onset $/ 7 /$. The growth of the tearing instability requires a strongly concentrated, intense current, in addition to some dissipation mechanism to release the free energy. If the current sheet thickness is of the order of 
the electron Larmor radius, chaotic scattering of the thermal electron population has been suggested to trigger the collisionless ion tearing instability $/ 8 /$.

The changes in the magnetospheric configuration from dipolar to taillike as the level of magnetic activity increases have been recognized for a long time $/ 9,10 /$. Several recent case studies suggest that a thin and intense current sheet is formed close to the inner edge of the plasma sheet with a minimum thickness at the electron gyroradius scale $/ 11,12,13 /$. A temporally-evolving magnetic field model with several free parameters, such as the thickness of the cross-tail current sheet and intensity of the current, was devised to follow the development of the magnetotail prior to the substorm onset. The magnetic field configuration and current sheet profile in each case were determined by finding a best fit with observations from several suitably located spacecraft. The model gives the global magnetic field configuration at each time, although the accuracy of the model is best close to the locations where the observations were made.

Comparison of the global model results with local methods to determine the current sheet characteristics provides an important test for the model. In this paper, the current sheet thickness during the growth phase of the CDAW-6 substorm (22 March 1979, onset at 1054 UT) is obtained from the global model, and compared with an earlier local calculation by McPherron et al. /1/. The tail stability towards ion tearing at substorm onset is estimated from the degree of electron pitch-angle scattering $/ 8 /$.

\section{CURRENT SHEET THICKNESS ESTIMATES}

On 22 March at 1100 UT, several spacecraft were favorably located for detection of the substorm development. GOES-3 at geostationary orbit and the ISEE-1 and -2 spacecraft were aligned along the same meridian, which was also close to the substorm onset meridian, and the solar wind conditions were monitored by the IMP- 8 satellite in the dusk sector (see $/ 14 /$, for an event overview).

The substorm growth phase started $\sim 1020 \mathrm{UT}$, shortly after the southward turning of the IMF $B_{Z}$ at 1010 UT. The IMF stayed strongly southward until about 1120 UT. Figure 1 shows the magnetic field observations from GOES-3 and the ISEE-1 and -2 spacecraft for this event. At geostationary orbit, the growth phase was seen as strong enhancement of $B_{X}$ and a reduction in $B_{Z}$, leading to a highly tail-like field configuration. At substorm onset (1054 UT) $B_{X}$ reduced, which was followed by an increase in $B_{Z}$; the field returned to its pre-growth phase configuration in about 10 minutes. The ISEE-1 and -2 spacecraft at $\sim 13 R_{E}$ distance were located very close to the current sheet early in the growth phase. The current sheet passed by the satellites a few times, as shown by the changing sign of the $B_{X}$-component. Finally, at about 1034 UT ISEE-1, and ISEE-2 a few minutes later, crossed to the northern side of the current sheet, and remained there until after the substorm onset. The data suggest that the current sheet was intensifying and simultaneously moving away from the spacecraft. Shortly after the substorm onset, ISEE-2 crossed the current sheet, and the two spacecraft were on opposite sides of the current sheet for almost 10 minutes. The strong fields observed by the two closely spaced spacecraft on each side of the current sheet imply that the current sheet was extremely thin and intense $/ 1 /$.

The development of the current sheet at the ISEE location has been previously studied by McPherron et al. $/ 1 /$. Using principal axis analysis they concluded that the current sheet was not at its nominal location along the Sun-Earth line, but was tilted $10^{\circ}$ below the equatorial plane and rotated $15^{\circ}$ in the $X-Y$ plane. McPherron et al. /1/ calculated the thickness and intensity of the current sheet using magnetic field data from ISEE-1 and -2 and a three-parameter model. At substorm onset they obtained a current sheet thickness that was less than the vertical separation of the spacecraft (about $3000 \mathrm{~km}$ ), and deduced a minimum value of $\sim 400 \mathrm{~km}$ shortly after the substorm onset (Figure 3).

We have modified the Tsyganenko $1989 / 15 /$ field model (referred to as T89) to describe the 


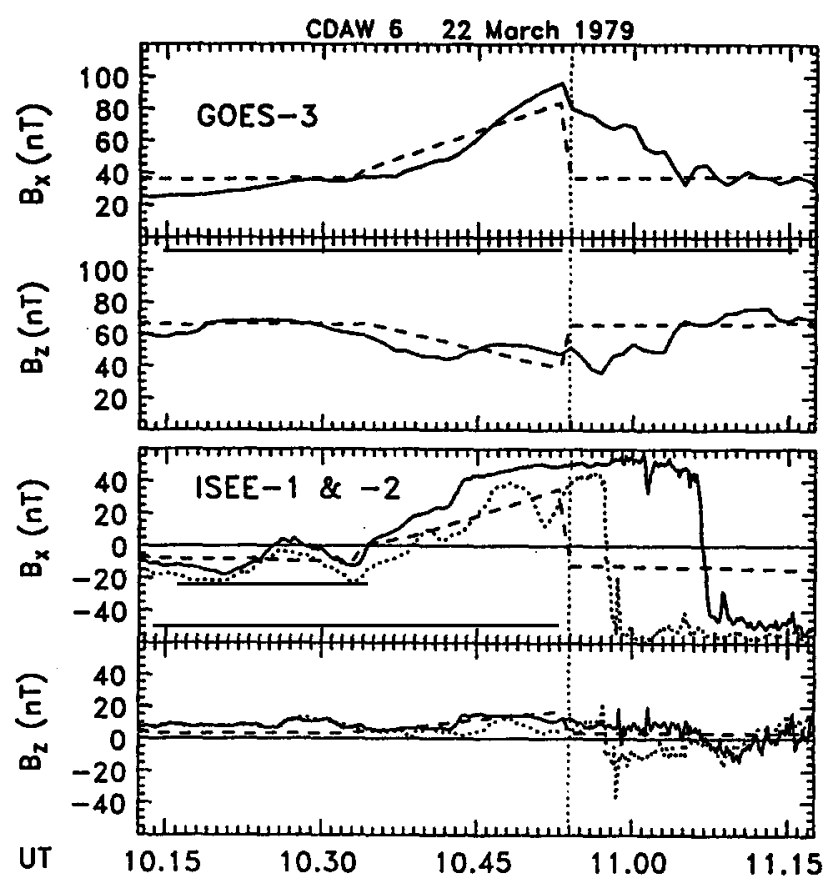

Fig. 1. Magnetic field for the GOES-3 and ISEE-1 (solid line) and -2 (dotted line) spacecraft in GSM-coordinates. The dashed lines show the model results. Times before $1034 \mathrm{UT}$ and after $1054 \mathrm{UT}$ are calculated using the T89 model $(K p=3)$. During the period 1034-1054 UT the tail current sheet is rotated away from the Sun-Earth line, the currents are intensified and the current sheet is thinned. The model parameter values are determined using 1-min averages of the data and a least squares fitting procedure.

temporal evolution of the global field configuration during the growth phase. The total cross-tail current is intensified and the tail current sheet is thinned during the growth phase. An additional current system describing the strong substorm-associated current system has been added to the T89 model. Also this current intensified during the growth phase (for details of the model see /13/). The parameter values (e.g., current sheet thickness, current intensity, radial location and intensity of the substorm-associated current system) were determined by a least-squares method using 1-min. averages of the magnetic field observations during 1034 - 1054 UT from the GOES-3 and ISEE-1 spacecraft.

After 1034 UT the current sheet determined from the T89 model is located above the ISEE1 location $\left(B_{X, M O D E L}<0\right)$, whereas the data indicate that the current sheet was below the spacecraft $\left(B_{X, M E A S U R E D}>0\right)$. Thus, the development of the currents during this event cannot be modeled by simple intensification of the model currents as described above; the deviation in the current sheet location from its statistical position has to be included in the model. We have rotated the tail field starting with the current sheet at the ISEE-1 location at 1034 UT, when the data indicate a current sheet crossing. Between 1034 UT and 1054 UT the current sheet is further rotated away from the ISEE-l location such that the rotation angle increases linearly with time. We obtained an optimal fit to the data by choosing the maximum rotation angle to be $16^{\circ}$ at 1054 $\mathrm{UT}$, in good agreement with the results of $\mathrm{McPherron}$ et al. $/ 1 /$. The field lines in the midnight meridian are shown for the T89 model with $K p=3$ (Figure $2 \mathrm{a}$ ), and the rotated and stretched tail field at the time of the substorm onset (Figure $2 \mathrm{~b}$ ). Note how the intense current stretches the field lines to a much more taillike configuration.

The model results at the GOES-3 and ISEE-1 locations are shown by the dashed lines in Figure 
1. Note that only the period between $1034 \mathrm{UT}$ and $1054 \mathrm{UT}$ was used in the temporally evolving model, before 1034 UT and after 1054 UT the field was computed using the T89 model $(K p=3)$. The model field increase is partly due to the motion of the current sheet away from the spacecraft location, and partly due to the increasing currents. The current was strongly intensified, at the ISEE-distance the current intensity increased from about 60 to $120 \mathrm{~mA} / \mathrm{m}$ during the growth phase.
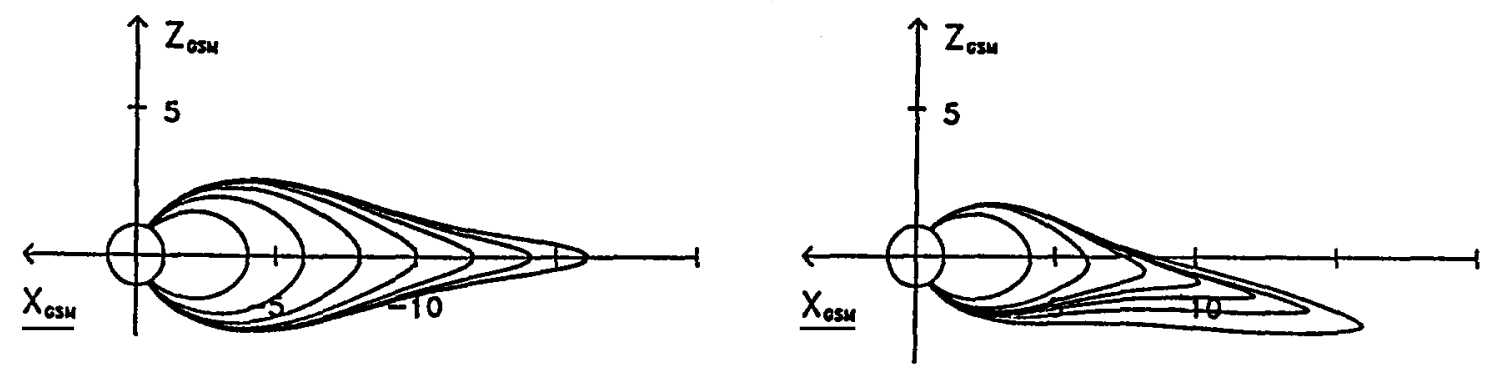

Fig. 2. Magnetic field lines calculated using the T89 model $(K p=3)$ describing the situation at the beginning of the growth phase. b) Magnetic field lines calculated using the modified model including the strong field stretching and rotation of the tail current sheet. The situation corresponds to maximal rotation and current intensification at substorm onset (1054 UT).

Figure 3 shows the current sheet thickness calculated from the global model at the ISEE-1 location, and the current sheet thickness estimated by McPherron et al. /1/. According to the McPherron et al. calculation, the plasma sheet rapidly thinned from about $6 R_{E}$ to less than $2 R_{E}$ during the early part of the growth phase. This value agrees well with the current sheet thickness parameter determined from the T89 model at 1035 UT. The local current sheet thickness calculation shows a wavy perturbation in the thickness superposed on the overall thinning of the current sheet $/ 1 /$. This may be due to either motion of the entire current sheet or wave-like disturbances in the current intensity at the ISEE-distance. Of course, these details cannot be reproduced by the global model. However, the average development of the current sheet thickness is in good agreement with the local model results.

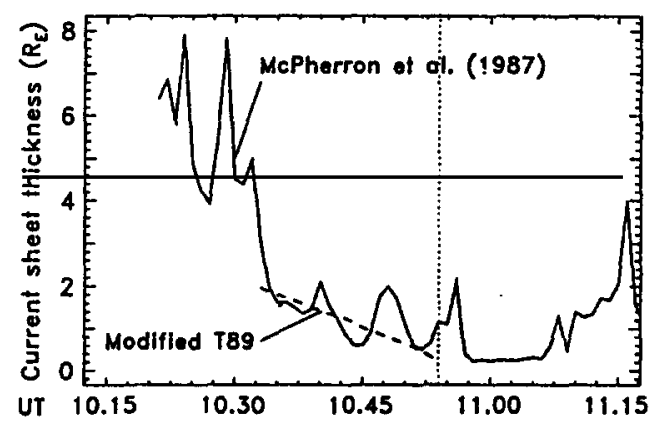

Fig. 3. Comparison of the current sheet thicknesses given by the modified Tsyganenko model (dashed line) and the local calculation by McPherron et al. /1/ (solid line).

One of the problems in global magnetic field modeling is the lack of direct observations of the current sheet thickness. Whereas the current intensity can be relatively accurately monitored by spacecraft away from the current sheet, the current sheet thickness obtained from the global model is an inferred quantity that may also reflect model properties. The good correspondence between the local estimate of the current sheet thickness using two spacecraft by McPherron et al. $/ 1 /$ and the global field model developed in this paper shows that the global modeling method can produce 
quite accurate results. Of course, the accuracy is greatest close to the locations of the spacecraft used in the least squares fit. These complementary methods gave very similar values as well for the current sheet thickness as for the current sheet location (rotation angle) and the total current intensity.

\section{ELECTRON CHAOTIZATION AT SUBSTORM ONSET}

Pitch-angle scattering of thermal electrons has been suggested to destabilize the ion tearing instability at substorm onset $/ 8 /$, leading to the disruption of the cross-tail current and diversion of the current into the ionosphere as the substorm current wedge. We have used the global magnetic field model to study electron scattering in the equatorial current sheet. Electrons are effectively pitch-angle scattered when the magnetic field line curvature radius becomes comparable to the local particle Larmor radius, $R_{C} / \rho_{e}<10$. Figure 4 a shows the regions in the equatorial current sheet where the $1 \mathrm{keV}$ electrons are scattered close to the substorm onset. At the beginning of the modeling period at $1034 \mathrm{UT}$, the thermal electron population was adiabatic throughout the inner magnetosphere. Starting at 1046 UT a small region of weak electron scattering developed in the tail centered around the midnight meridian at a distance of $\sim 11 R_{E}$. At the time of the substorm onset this region had expanded Earthward to $\sim 8 R_{E}$, and the scattering became stronger.

Figure $4 \mathrm{~b}$ shows the electron spectra. from the LEPEDEA-instrument onboard ISEE-1. The perpendicular direction and two parallel directions are shown separately. During the growth phase, the electrons are anisotropic below about $1 \mathrm{keV}$. Note that the electron temperature was $\sim 1.5 \mathrm{keV}$ at the beginning of the growth phase, and $\sim 0.5 \mathrm{keV}$ prior to the substorm onset, implying that a large portion of the electron population was below the instrument threshold. The isotropization of the low-energy electron at 1045-1053 UT suggests that an effective pitch-angle scattering mechanism is active in that part of the tail. Within the temporal resolution of the spectra $(8 \mathrm{~min})$, this occurs simultaneously with the appearance of chaos as determined from the global field podel.
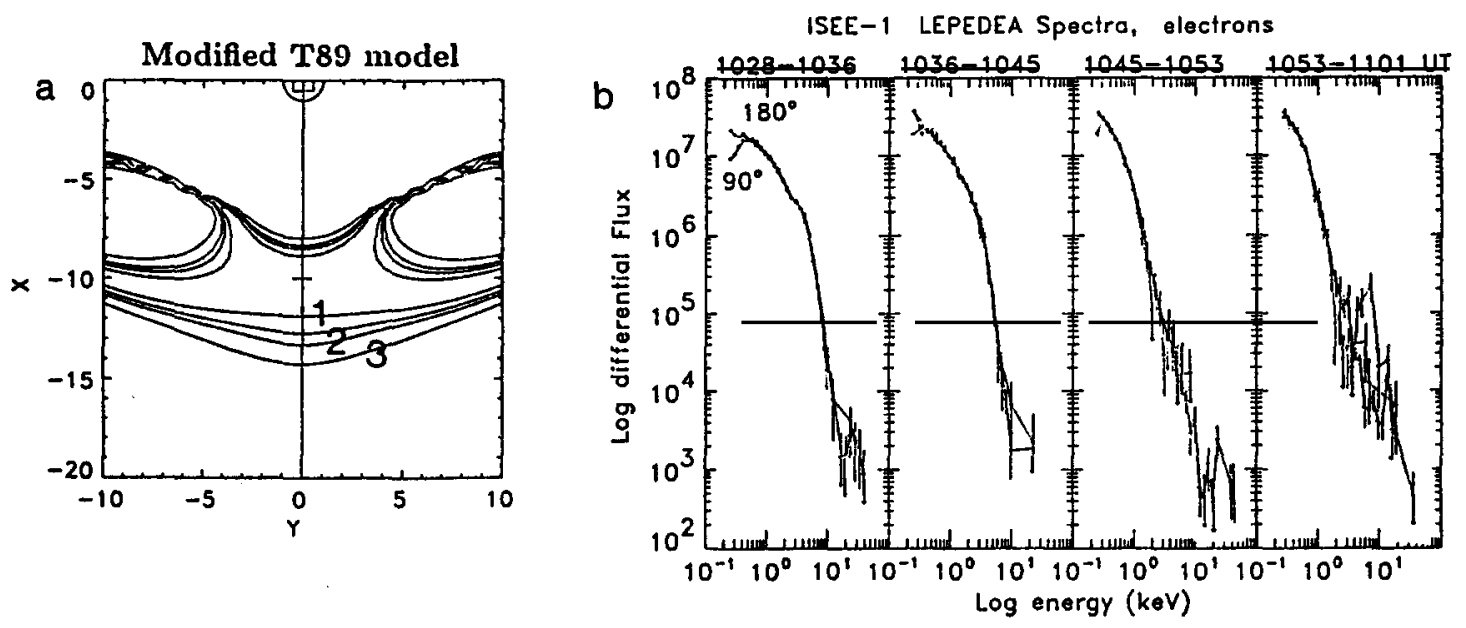

Fig. 4. a) Contour plots showing curyes of constant $\sqrt{R_{C} / \rho_{e}}$ at the current sheet at 1054 UT. The values $1,1.6,2$, and 3 are drawn. b) Electron spectra from the LEPEDEA instrument onboard ISEE- 1 for two parallel directions (pitch-angles $0^{\circ}-30^{\circ}$ and $150^{\circ}-$ $180^{\circ}$ ) and perpendicular to the field (pitch-angles $75^{\circ}-105^{\circ}$ ). The differential fluxes are shown as a function of energy in a $\log -\log$ scale. The parallel component is denoted by $" 180^{\circ}$, and the perpendicular component by " $90^{\circ} "$.

In this case, the scattering of the electrons is suggested both by the isotropization of the electron spectra and the global field model. Furthermore, the chaotic region is close to the region where the 
neutral line has been interpreted to form $/ 14 /$. These results are in good agreement with earlier modeling results of the current sheet development during strong substorm growth phases $/ 12,13 /$.

\section{DISCUSSION}

The near-Earth neutral line model postulates that the large-scale reconfiguration is a consequence of the onset of reconnection at a newly formed neutral line in the near-Earth tail $/ 16,17 /$. Baker and McPherron /18/ recently extended this model by suggesting that slow reconnection on closed field lines is initiated already during the late growth phase. This would redistribute the current within the current sheet, leading to a concentration of the current in a relatively narrow region. This would account for the strongly stretched field configurations observed close to the inner edge of the plasma sheet $/ 3,12 /$. In this scenario, substorm onset would follow when reconnection has proceeded to open field lines, when the larger Alfvén speed in the lobe would strongly increase the reconnection rate.

On the global scale, MHD simulations can reproduce the large scale signatures of substorms, such as field dipolarization, fast flows, and the ejection of plasmoids. In these models, resistive tearing instability develops as the current density increases in the field reversal region $/ 19 /$. The simulation results show that the different aspects of reconnection do not appear simultaneously at substorm onset /20/: The fast flows and the dipolarization of the field often appear prior to the X-line formation. Moreover, the current is first diverted within the current sheet in the unstable region, before the field-aligned currents associated with the substorm current wedge form. These results support the scenario proposed by Baker and McPherron /18/. In addition, the global model results of the intensification of the cross-tail current and electron chaotization few minutes before the onset of the global instability reported by Pulkkinen et al. /13/ and in this paper would provide the necessary dissipation mechanism.

One of the unresolved problems of the current sheet evolution is what particles carry the growthphase associated current. In the time stationary approximation, the perpendicular current has two terms, the pressure gradient term and the pressure anisotropy term inversely proportional to the field line radius of curvature. Mitchell et al. /21/ suggested that the anisotropic electron population accounts for much of the intensifying current during the growth phase, as the current increases due to the decreasing field line radius of curvature.

In the CDAW-6 event, the parallel electron pressure was greater during the growth phase $\left(P_{\|}-P_{\perp} \simeq\right.$ $3 \cdot 10^{-11} \mathrm{~Pa}$ ), until the anisotropy vanished close to the substorm onset. Thus the electron current added to the intensifying cross-tail current, but its contribution was not very large. On the other hand, the ion pressure anisotropy was an order of magnitude larger, but the anisotropy was variable in direction. The perpendicular pressure was larger 1003-1037 UT, after which the parallel pressure was larger (1037-1054 UT). The perpendicular pressure was again greater during the period 10541103 UT. After the substorm onset the parallel component increased strongly. As the direction of the anisotropy current varied, it cannot support the strong current in the positive Y-direction. However, because the current due to the anisotropy flows in a very narrow region close to the center of the current sheet, the 8-min time resolution of the data may affect this conclusion.

\section{CONCLUSIONS}

The development of a very thin and intense current sheet during the growth phase of a substorm has been studied using the CDAW- 6 observations from geostationary orbit and the near-Earth tail. The observed current sheet thinning has been modeled using a global magnetic field model. The model results agree well with previous local calculations of the current sheet development by McPherron et al. $/ 1 /$.

The plasma electron and magnetic field data were used to estimate the stability of the tail towards the ion tearing instability. The low-energy electrons that were anisotropic during much of the 
growth phase, were isotropized before the substorm onset. The global field model developed here suggests that the current sheet was thin enough to scatter thermal electrons. Both results support the scenario that the substorm onset is initiated by the tearing mode which is destabilized by pitch-angle scattering of thermal electrons $/ 8 /$.

\section{REFERENCES}

1. McPherron, R. L., A. Nishida, and C. T. Russell, in Y. Kamide and R. A. Wolf (eds): Quantitative modeling of magnetosphere-ionosphere coupling processes, Kyoto, Japan, 1987.

2. Bame, S. J., J. R. Asbridge, H. E. Felthauser, E. W. Hones, Jr., and I. B. Strong, J. Geophys. Res., 72, 113, 1967.

3. Baker, D. N., P. R. Higbie, E. W. Hones, Jr., and R. D. Belian, J. Geophys. Res., 83, 4863, 1978.

4. Fairfield, D. H., E. W. Hones, Jr., and C.-I. Meng, J. Geophys. Res., 86, 11189, 1981.

5. Hones, E. W., Jr., T. Pytte, and H. I. West, Jr., J. Geophys. Res., 89, 5471, 1984.

6. Jacquey, C., J. A. Sauvaud, and J. Dandouras,Geophys. Res. Lett., 18, 389, 1991.

7. Schindler, K., J. Geophys. Res., 79, 2803, 1974.

8. Büchner, J. and L. M. Zelenyi, J. Geophys. vRes., 92, 13456, 1987.

9. West, H. I., Jr., R. M. Buck, and M. G. Kivelson, J. Geophys. Res., 83, 3805, 1978.

10. Lui, A. T. Y., Geophys. Res. Lett., 5, 853, 1978.

11. Sergeev, V. A., P. Tanskanen, K. Mursula, A. Korth, and R. C. Elphic, J. Geophys. Res., 95, $3819,1990$.

12. Pulkkinen, T. I., D. N. Baker, D. H. Fairfield, R. J. Pellinen, J. S. Murphree, R. D. Elphinstone, R. L. McPherron, J. F. Fennell, R. E. Lopez, and T. Nagai, Geophys. Res. Lett., 18, 1963, 1991.

13. Pulkkinen, T. I., D. N. Baker, R. J. Pellinen, J. Büchner, H. E. J. Koskinen, R. E. Lopez, R. L. Dyson, and L. A. Frank, Particle scattering and current sheet stability in the geomagnetic tail during the substorm growth phase, J. Geophys. Res., in press, 1992.

14. McPherron, R. L. and R. H. Manka, J. Geophys. Res., 90, 1175, 1985.

15. Tsyganenko, N. A.,Planet. Space Sci., 37, 5, 1989.

16. Hones, E. W., Jr., Dynamics of the magnetosphere, 545, D. Reidel, Dordrecht, Holland, 1979.

17. McPherron, R. L., C. T. Russell, and M. P. Aubry, J. Geophys. Res., 78, 3131, 1973.

18. Baker, D. N. and R. L. McPherron, J. Geophys. Res., 95, 6591, 1990.

19. Birn, J. and E. W. Hones, Jr., J. Geophys. Res., 86, 6802, 1981.

20. Birn, J. and M. Hesse, J. Geophys. Res., 96, 1611, 1991

21. Mitchell, D. G., D. J. Williams, C. Y. Huang, L. A. Frank, and C. T. Russell, Geophys. Res. Lett., $l 7,583,1990$. 